\title{
International Workshop on Open and Original Problems in Software Language Engineering
}

\author{
Anya Helene Bagge ${ }^{\star}$ and Vadim Zaytsev ${ }^{\dagger}$ \\ ${ }^{\star}$ Bergen Language Design Laboratory (BLDL), University of Bergen, Norway \\ ${ }^{\dagger}$ Centrum Wiskunde \& Informatica and Universiteit van Amsterdam, The Netherlands
}

The second international workshop on Open and Original Problems in Software Language Engineering (OOPSLE'14) follows the first one held at WCRE 2013 in Koblenz. It is meant to be a discussion-oriented and collaborative forum for formulating and addressing with open, unsolved and unsolvable problems in software language engineering (SLE), which is a research domain of systematic, disciplined and measurable approaches of development, evolution and maintenance of artificial languages used in software development. OOPSLE aims to serve as a think tank for identifying and formulating challenges in the software language engineering field these challenges could be addressed later at venues like SLE, MODELS, CSMR, WCRE, ICSM and others.

The field covered by the workshop, revolves around "software languages" - all kinds of artificial languages used in software development: for programming, markup, prettyprinting, modelling, data description, formal specification, evolution, etc. Software language engineering is a relatively new research domain of systematic, disciplined and measurable approaches of development, evolution and maintenance of such languages. Many concerns of software language engineering are acknowledged by reverse and forward software engineers: robust parsing of language cocktails, fact extraction from heterogeneous codebases, tool interfaces and interoperability, renovation of legacy systems, static and dynamic code analysis, language feature usage analysis, mining repositories and chrestomathies, library versioning and wrapping, etc.

Some research fields have a list of acknowledged open problems slowly addressed by the community: Hilbert's problems ${ }^{1}$, the POPLmark Challenge ${ }^{2}$, a list of open problems in Boolean grammars ${ }^{3}$, etc - the SLE field has not yet produced one. This workshop is meant to expose hidden expertise in coping with unsolvable or unsolved problems which commonly remain unexposed in academic publications. OOPSLE aims to serve as a think tank in selecting candidates for the open problem list, as well as other kinds of unconventional questions and definitions that do not necessarily have clear answers or solutions, thus facilitating the exposure of dark data. We also plan to formulate promising language-related challenges to organise in the future. Beside the abovementioned POPLmark Challenge which can also be seen as a collection of benchmarks, there

\footnotetext{
${ }^{1}$ http://aleph0.clarku.edu/ djoyce/hilbert/

${ }^{2} \mathrm{http}: / /$ www.seas.upenn.edu/ plclub/poplmark/

${ }^{3} \mathrm{http}: / /$ users.utu.fi/aleokh/boolean/nine_open_problems.html
}

have been many more contests, challenges and competitions related to software language engineering: LDTA Tool Challenge held at the LDTA workshop in $2011^{4}$, CodeGenerationaffiliated Language Workbench Challenge ${ }^{5}$ held yearly since 2011, Transformation Tool Contest held six times since $2007^{6}$, Rewrite Engines Competition held three times in 2006, 2008 and 2010 at $\mathrm{WRLA}^{7}$, PLT Games held monthly since $2012^{8}$.

Before the workshop OOPSLE participants are encouraged to submit position papers up to 4 pages in length, sketching an open or original problem, idea or challenge. The submissions are screened by the workshop chairs, who will select papers based on potential for discussion and interest to the community, as well as the clarity of presentation and motivation - OOPSLE is not a mini-conference, and therefore it is not necessary for the work to be conclusive yet. The papers will be posted online prior to the workshop, so the participants have the opportunity to read them in advance.

At the workshop, two keynote lectures (an academic one and an industrial one) will be given to provide an overview of some existing problems in the field of language engineering and to serve as a starting point for discussion during the rest of the day. Each accepted position paper will also be presented as a brief summary of its main idea and a set of open questions to be discussed with the audience. Presenters will ask for input on how to proceed with experiments, validation or refinement of their ideas, collect opinions on the presented definitions, share similar experience. We expect the participants to be friendly but inquisitive, and ask hard questions back that may lead to deepening the initially presented insights. The workshop is planned to have short presentations and long discussions to stimulate direct collaboration afterwards.

After the workshop, all workshop participants will be invited to submit a full paper to a special issue of the Electronic Communications of the EASST, an open access peer-reviewed journal. Journal submissions will undergo peer review by the members of the program committee consisting of researchers in software language engineering and reverse engineering.

An extensive yet non-exhaustive collection of topics encouraged for investigation for workshop participants, can be found at http://oopsle.github.io.

\footnotetext{
${ }^{4}$ http://ldta.info/tool.html

${ }^{5} \mathrm{http}: / /$ www.languageworkbenches.net

${ }^{6}$ http://planet-sl.org/ttc2013/

${ }^{7}$ http://www.lcc.uma.es/ duran/rewriting_competition/

${ }^{8}$ http://www.pltgames.com
} 